\title{
MRI of Lateral Skull Base Lesions
}

\author{
Deepak P Patkar, Rama Yanamandala, Mukesh Pardhi, Sona A Pungavkar \\ Vishal Gaikwad, Sudarshan Vijay Pawar \\ Department of MRI, Dr Balabhai Nanavati Hospital, Vile Parle (West), Mumbai, Maharashtra, India
}

Correspondence: Rama Yanamandala, Department of MRI, Dr Balabhai Nanavati Hospital, SV Road, Vile Parle (West), Mumbai400056, Maharashtra, India, Phone: 91-22-26134455, 91-22-26182712, Fax: 91-22-26119363, e-mail: yrama555@ gmail.com

\begin{abstract}
This article reviews the role of magnetic resonance imaging (MRI) in the evaluation of lateral skull base lesions. Due to superior soft tissue resolution and multiplanar capability, MRI provides accurate information and exquisite anatomical detail. Thus, it guides the surgeon in proper preoperative planning regarding the approach of a lesion. MRI is also useful in post-treatment follow-up to assess the therapeutic response and to identify potential complications. We discuss the relevant anatomy, indications of MRI and MR pulse sequences used in the diagnosis of lesions of lateral skull base. Characteristic MRI findings of various lesions which help to arrive at a specific diagnosis as well as pitfalls of MRI which may confound the diagnosis are described. Newer MR pulse sequences enable image-guided surgery, which assist the surgeon intraoperatively, are briefly discussed. In summary, this article emphasizes the role of MRI in providing a specific answer to a clinical problem and its ability to guide the clinician for better management of patients.
\end{abstract}

Keywords: Computed tomography (CT), Magnetic resonance imaging (MRI), Cerebellopontine angle (CPA), Internal auditory canal (IAC), Cerebrospinal fluid (CSF).

\section{INTRODUCTION}

Lateral skull base is not a precisely defined area. It corresponds to the region of temporal bone. ${ }^{1,2}$ Numerous foramina and channels carrying neurovascul ar structures are enclosed in the skull base. M any vital structures are present within the skull base. Thorough knowledge of the anatomy is important in assessing a pathology. Modern imaging techniques with great precision help the surgeon to map the complex anatomy of skull base and to identify the pathologies. Determination of the position of apparent origin of a pathology helps to narrow the differential diagnosis. The characteristic imaging findings help to further narrow the list. Imaging provides the exact knowledge of anatomical landmarks and relation of adjacent structures to the pathology which are crucial to decide the management and the surgical feasibility of a lesion.

Computed tomography (CT) and magnetic resonance imaging (M RI) are appropriate for the evaluation of skull base. MRI being the modality with better soft tissue resolution permits precise assessment of morphology of a lesion and its relation with surrounding structures. MRI is also superior in the evaluation of vascular invasion and intracranial extension. M RI is clearly the modal ity of choice for retrocochlear pathologies. MRI is a problem-solving modality in many situations where $\mathrm{CT}$ and other modal ities cannot give the required information. Newer M R pulse sequences are highly sensitive in detection of pathologies and al so hel p to give a specific diagnosis. A Iso, the extensive development of neuronavigational techniques provide valuable information to surgeon to safely and effectively remove even the most complex lesions with minimal morbidity. Though the bony details and air spaces are best evaluated by CT, MRI can give useful information about these structures due to signal from fluid or mass in diseased states. In some situations, both these modalities complementary. ${ }^{3}$ The relevant anatomy, M R sequences used to evaluate various pathologies, pitfalls due to normal variants and artefacts, and disadvantages of MRI are addressed in respective sections.

This article presents lateral skull base lesions under following sections:

Lesions involving temporal bone, jugular foramen, cerebellopontine angle (CPA) and internal auditory canal (IAC). Facial nerve lesions are discussed in detail in a separate section.

\section{TEMPORAL BONE LESIONS}

Temporal bone lesions can be divided on the basis of etiology into congenital, inflammatory, traumatic and neoplastic lesions. Lesions of petrous apex are considered in a separate section.

\section{Congenital Lesions}

M RI is now being increasingly used to image patients with congenital inner ear anamolies. Pathologies of membranous labyrinth and internal auditory canal (IAC) are well demonstrated on MRI. A brief discussion on the role of $M R I$ in common congeital anamolies is considered here. The reader is referred somewhere for detailed description of congenital inner ear anamolies. ${ }^{4-6}$ 
Thin section ( $<1 \mathrm{~mm}$ thickness) heavily T2-weighted gradient echo or spin echo images are sensitive in detecting inner ear anamolies. These sequences provide images with bright intralabyrinthine fluid surrounded by dark bone and nerves. 3D sequences can be used to acquire images in multiple planes, and the use of multiplanar reconstructions and maximum intensity projections help to eval uate complex anamolies in detail. High resolution M RI well-demonstrates the divisions of vestibulocochlear nerve separately within the IAC and accurate assessment of their diameters can be made (Fig. 1). Thus, it helps to diagnose aplasia or hypoplasia of individual nerves. Gupta et al have used the term cochlear nerve deficiency for both absent and hypoplastic cochlear nerves ${ }^{5}$ (Fig. 2).

Studies showed that cochlear nerve is larger than either superior or inferior vestibular nerves in $90 \%$ of individuals and if of equal size or larger than facial nerve in $64 \%$ of cases. $^{7,8}$ Cochlear nerve size correlates with the outcome of

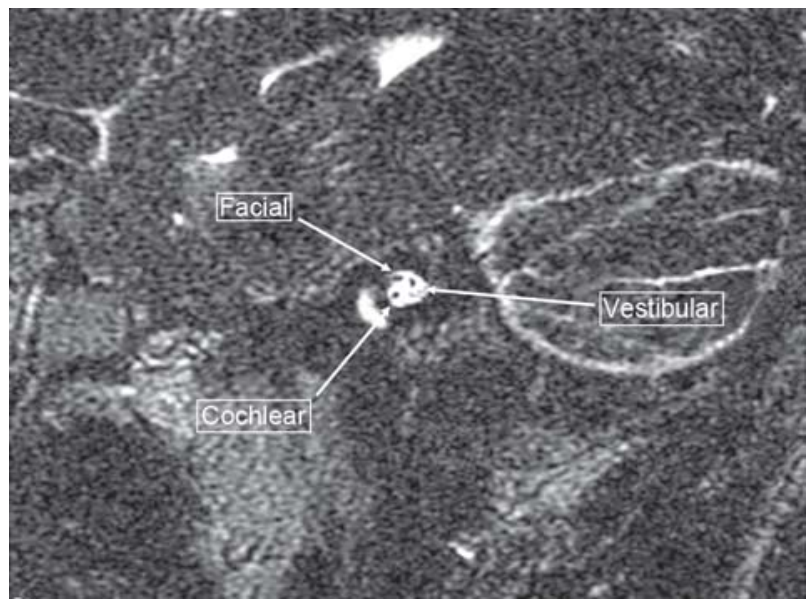

Fig. 1: Oblique sagittal CISS image shows all the four nerves in the IAC. Facial nerve is seen in the anterosuperior, cochlear nerve is seen in the anteroinferior, superior and inferior vestibular nerves in the posterosuperior and posteroinferior quadrants

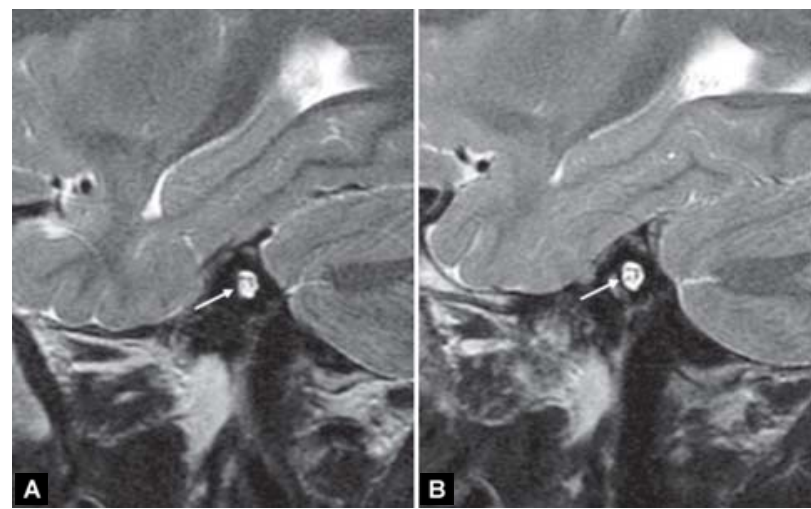

Figs 2A and B: Congenital cochlear nerve deficiency. Oblique sagitta T2-weighted images. On the affected side (A), Cochlear nerve in the anteroinferior quadrant is smaller in caliber (white arrow) compared to the normal side. Image of the unaffected side (B) shows cochlear nerve of normal size cochlear implantation. ${ }^{9}$ H owever, even an appreciably thin cochlear nerve may be capable of effectively transmitting impulses in few cases. ${ }^{5,10}$ Therefore, a small nerve is only a rel ative contraindication to cochlear implantation. In patients where the cochlear nerve is not seen on M RI, it is possible that it is so thin that it is beyond the resolution of MRI scanner or it may be that the cochlear nerve fibers traverse along with fibers of the vestibular nerve. Thus, these patients should undergo a hearing aid trial and evaluation by expert audiologists is suggested to determine their candidacy for cochlear implantation. Intracochlear electrical stimulation may be a valuable test to perform in such patients before surgery. ${ }^{5}$

In vestibular aqueduct syndrome, most common cause of congenital sensorineural hearing loss, a large endolymphatic duct or sac is seen at MR imaging. ${ }^{11}$ T2-weighted images show the hyperintense, enlarged fluid filled endolymphatic duct and sac surrounded by hypointense bony structures. The full extension of the endolymphatic sac into the posterior fossa, which is a major component of the enlarged endolymphatic structure is seen on MRI. This is often not visible on CT. MR images, sometimes, show a thin dural lining between the endolymphatic sac and cerebrospinal fluid (CSF), thus help to follow the endolymphatic sac from vestibule into posterior fossa. ${ }^{6}$ Also, MRI can demonstrate fibrous obliteration of the duct and sac which show a lower signal intensity than CSF or intralabyrinthine fluid. ${ }^{12}$

Scala vestibuli and scala tympani can be shown as distinct structures on M RI. ${ }^{13}$ Even the minute structures, such as interscalar septum and osseous spiral Iamina, can be visualized with the resolution provided by the newer M R scanners. Fibrous obliteration of scala tympani and scala vestibuli can be seen on M RI.

\section{Inflammatory Lesions}

M RI plays a crucial role in identifying potential complications of inflammatory conditions of temporal bone which can be considered based on anatomic sites: External ear, middle ear and mastoid and inner ear.

\section{Middle Ear and Mastoid}

Infections of middle ear and mastoid can be acute and chronic. M RI is indicated in imaging of some of the sequelae of acute and chronic otomastoiditis.

Important complications of acute otomastoiditis, wherein $M R I$ is considered include dural sinus thrombosis, layrinthitis and petrous apicitis.

M RI is ideal for diagnosis and foll ow-up of patients with dural sinus thrombosis. ${ }^{14} \mathrm{M} \mathrm{RI}$ venography is considered to be state of the art investigation in evaluation of these patients. M RI findings can be direct or indirect. ${ }^{4}$ Demonstration of 
thrombus as filling defect within the sinus is the direct evidence of thrombosis. This can be appreciated on T2-weighted and proton density weighted spin echo images in the deoxyhemoglobin stage. Indirect findings include absence of expected normal flow void in the venous sinuses on spin echo sequences and flow related enhancement on gradient echo images. False-positive diagnosis of sinus thrombosis can result due to vagaries in flow phenomena and the varying signal intensity of blood products. ${ }^{15}$ Presence of aberrant arachnoid granulation which may appear as filling defect also poses diagnostic difficulty. Diffusion and perfusion weighted $M R$ images provide information regarding physiologic state of the brain after dural sinus thrombosis. This allows triage of patients between conservative therapy and microcatheter-directed thrombolysis. ${ }^{16}$

Spread of exotoxins to labyrinth results in labyrinthitis. $M R$ imaging reveals enhancement of the membranous labyrinth on contrast enhanced T1-weighted M R images. Pathologic enhancement of the meninges at the periphery of the erosive change of the petrous apex is seen in petrositis, along with possible extension to M eckel's cave involving gasserian ganglion ${ }^{4,17}$ Important sequelae of chronic otitis media include granulation tissue and chol esteatoma. B oth these entities can be differentiated on contrast enhanced MRI.

Granulation tissue is probably the most common cause of middle ear debris. Granulation tissue shows intense enhancement as it is quite vascular. ${ }^{18}$ Cholesterol granuloma is a type of granulation tissue lined by fibrous connective tissue. ${ }^{19}$ It is the result of a hemorrhagic foreign body response elicited by cholesterol crystals. MR imaging is diagnostic as the lesion is hyperintense on all spin echo pulse sequences due to the presence of extracellular methemoglobin. Cholesterol granuloma at the petrous apex results in an expansile lesion, often referred to as giant cholesterol cyst. ${ }^{20}$

A quired cholesteatoma is unique to middle ear and is a classic entity considered in the context of chronic otitis media and a retrotympanic mass. ${ }^{4}$ They occur at any location in the middle ear cavity but are most commonly found in the posteromedial or anteromedial location. ${ }^{21} \mathrm{~A}$ number of theories have been described to explain these lesions, most favored being the invagination theory. It suggests that they devel op from retraction pockets in the tympanic membrane, which allow the keratin debris to accumulate and formation of cholesteatoma. Thus, it is simply skin growing in the wrong place consisting of keratinised stratified epithelium. Thus, cholesteatomas do not enhance on contrast enhanced M RI whereas granulation tissue enhances intensely due to presence of fibrous vascular connective tissue. This also helps to differentiate cholesteatoma from other retrotympanic masses, such as paraganglioma or schwannoma. ${ }^{4}$
Contrast enhanced M RI is thus highly accurate in separating cholesteatoma from other lesions.

Cholesteatomas may cause erosion of bone at many locations. Especially, ominous is involvement of tegmen tympani and sigmoid sinus plate as erosion at these sites could lead to epidural invasion. This can be a great potential for devel opment of meningitis, cerebritis, abscess or venous sinus thrombosis. Thus, M RI is indicted when bony erosion is detected involving these sites. Also, MRI can define an encephalocele extending through the eroded tegmen tympani. ${ }^{17}$

\section{Inner Ear}

L abyrinthitis is an inflammatory process of the membranous labyrinth due to an invading agent or it can be involved as a part of a systemic disease. It is the most commonly result of a viral infection. ${ }^{4}$ R egardless of etiology, contrast enhanced T1-weighted MR images reveal faint, diffuse, but ofen segmental enhancement of the normally nonenhancing, fluid filled spaces of the labyrinth. ${ }^{22}$ This is due to breakdown of the normal blood-endolymph barrier. Facial nerve enhancement may be associated. Precontrast T 1-weighted MR images are essential to distinguish enhancement of labyrinthitis from intralabyrinthine hemorrhage. In hemorrhage, high signal intensity of the labyrinth is due to the presence of methehemogl obin. ${ }^{23}$ Proteinaceous fluid also can result in high $\mathrm{T} 1$ signal intensity.

L abyrinthitis ossificans is the result of the repair process after a destructive or an inflammatory insult. It is divided into three stages. The acute phase is characterized by purulent and serofibrinous exudate in the perilymphatic spaces. Fibroblastic proliferation, resulting in fibrosis occurs in the second stage. The final stage is Iabyrinthine ossification. Fibrosis of the membranous labyrinth which may not be well-appreciated on $\mathrm{CT}$, is probably best seen on high resolution T2-weighted M R images as loss of normal

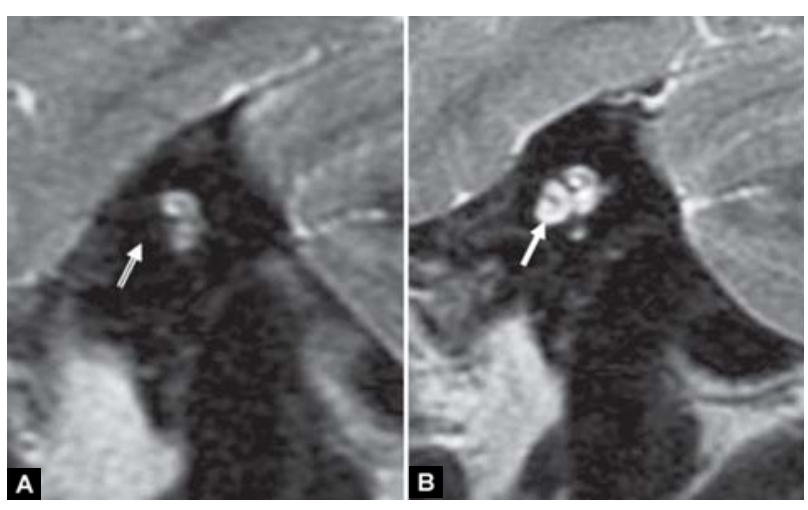

Figs $3 A$ and B: Labyrinthitis ossificans. Oblique sagittal T2-weighted images. There is loss of normal bright signal of the cochlea (A, open white arrow) due to replacement of CSF with new bone formation. Compare with normal cochlea (solid white arrow) on the opposite side (B). This patient had past history of viral infection 
hyperintensity. ${ }^{24,25}$ The ossific stage results in total "whiteout" of the membranous labyrinth. This leads to signal void in the labyrinth on T2-weighted images (Figs $3 A$ and $B$ ). Identification of this condition is of paramount importance when cochlear implantation is considered.

\section{External Ear}

Necrotizing external otitis is an aggressive osteomyelitis of the temporal bone. It begins as a focal area of ulceration and osteitis of external auditory canal, and then spreads to extensively involve adjacent bony and soft tissue structures. $M R I$ is superior to CT when intracranial extension of the pathology is suspected as the abnormalities of medullary bone of skull base and enhancement of dura and soft tissues is best appreciated ${ }^{26}$ (Figs 4A to C). However, M RI is not useful in evaluating the response to therapy, as the marrow changes caused by inflammation may take up to 6 months to return to normal. A combination of In-111 WBC and TC-99m SPECT may be the best imaging techniques to assess the therapeutic response in successfully treated patients than M RI, CT or gallium scans. ${ }^{27}$

\section{Traumatic Lesions}

Temporal bone fractures are classified into transverse, Iongitudinal or a mixed pattern. CT and M RI are complementary in the imaging eval uation of a patient with suspected temporal bone fracture. ${ }^{28} \mathrm{D}$ ural enhancement is seen on M RI even in patients with radiologically invisible fractures, believed to correlate with "microfractures", with associated microtears of adjacent dura. ${ }^{29,30} \mathrm{M} \mathrm{RI}$ also plays a crucial role in post-traumatic facial palsy as it helps in direct visualization of the nerve even when there is no demonstrable fracture on $\mathrm{CT}$. Intral abyrinthine hemorrhage causing sensorineural hearing loss is well-demonstrated on thin section noncontrast T1-weighted images. ${ }^{31,32}$

\section{Neoplastic Lesions}

Neoplastic lesions of temporal bone can be divided into those involving internal auditory canal and cerebel lopontine angle, jugular foramen, facial nerve and petrous apex lesions. A II these lesions are considered in respective sections.

\section{Cerebellopontine Angle and Internal Auditory Canal Lesions}

CPA and IAC lesions can be discussed together. Primary tumors of CPA include acoustic schwannoma, meningioma, epidermoid, arachnoid cyst, schwannoma of other cranial nerves (V , V II, IX X X, XI), lipoma, melanoma and dermoid. Other lesions include metastases and vascular lesions. Lesions from jugul ar foramen, clival and paraclival location, cerebellar, brainstem and petrous bone can secondarily involve CPA. The lesions can al so be grouped as intra-axial or extra-axial. L esions of brainstem and cerebellum are intraaxial in location and include ependymoma, medulloblastoma, choroid plexus papilloma, glioma. We stress on the most commonly encountered lesions in this region.

\section{Acoustic Schwannoma}

Schwannomas are benign, encapsulated neoplasms that originate in the nerve sheath. They account for 6 to $10 \%$ of all intracranial tumors and 60 to $90 \%$ of CPA tumors. ${ }^{33}$ They can arise within IAC, at the porus, or in the CPA cistern. ${ }^{34}$ Clinical manifestations depend on the size of the lesion. The most common symptoms are sensorineural hearing loss, tinnitus and disequilibrium due to pressure on the cochlear and vestibular divisions of acoustic nerve..$^{35,36}$

MRI is clearly the diagnostic modality of choice. Contrast-enhanced M RI has the highest negative predictive value and is the easiest examination to perform and interpret. ${ }^{37}$ High quality M R imaging contributes to early detection of these lesions which helps in hearing preservation. With MR imaging it is possible to determine the component of the vestibulocochlear nerve that is involved. ${ }^{7} \mathrm{M}$ RI can al so predict hearing preservation, when the labyrinthine signal is normal on constructive interference in the steady state (CISS) sequence or when there is absence of cochlear contrast enhancement. ${ }^{38,39}$ I maging findings are crucial in determining the surgical approach.

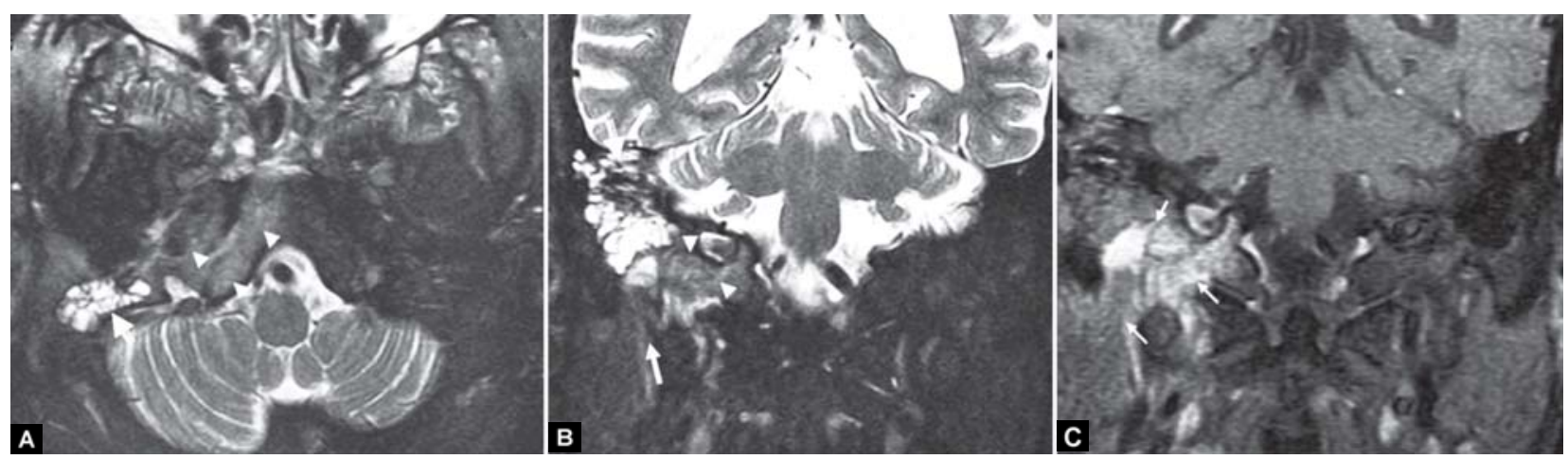

Figs 4A to C: Skull base osteomyelitis secondary to mastoiditis. (A) axial T2-weighted image, (B) coronal T2-weighted image, (C) coronal postcontrast T1-weighted image. Abnormal marrow signal intensity is seen involving petrous bone and clivus (arrow heads) along with altered fat signal in the adjacent soft tissues of medial skull base (solid white arrow) which show postcontrast enhancement (small white arrows). Hyperintense fluid signal is seen in the mastoid air cells suggestive of mastoiditis (open white arrows) 


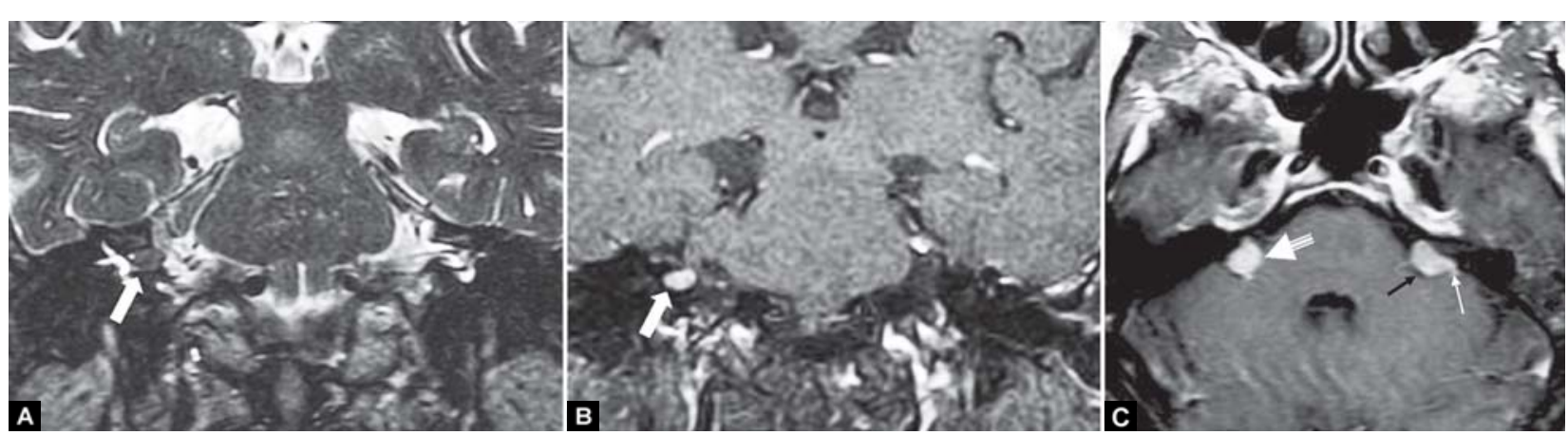

Figs 5A to C: Intracanalicular schwannoma. (A) coronal T2-weighted image and (B) coronal contrast-enhanced T1-weighted image. A welldefined mass lesion is in the intracanalicular location (solid white arrows). It shows homogenous postcontrast enhancement, (C) bilateral acoustic schwannomas in neurofibromatosis 2. Axial contrast-enhanced T1-weighted image. A well-defined spherical lesion with homogenous postcontrast enhancement is seen in the right cerebellopontine angle centered at porus acousticus (open white arrow). The mass on the left has spherical cisternal component (black arrow) with a small component extending into IAC (small white arrow). This appearance resembles an ice-cream cone or a mushroom

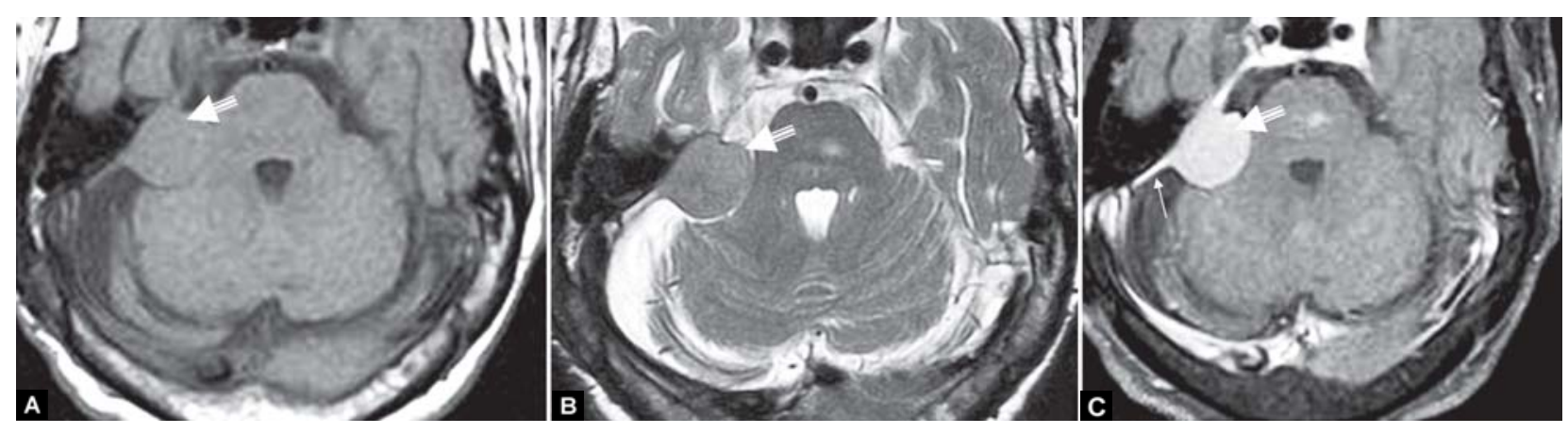

Figs 6A to C: Cerebellopontine angle meningioma. (A) axial T1-weighted image, (B) T2-weighted image (C) contrast-enhanced T1-weighted image. The tumor (open white arrows) is an extra-axial hemispherical mass lesion with broad base against posterior wall of petrous bone and obtuse bone tumor angle. It is isointense to gray matter on T1 and T2-weighted images and shows homogenous postcontrast enhancement. Enhancing dual tail is seen (small white arrow)

Intracanalicular tumors are small in size $(<1 \mathrm{~cm})$ and are usually cylindrical in shape ${ }^{40}$ (Figs $5 \mathrm{~A}$ and $\mathrm{B}$ ). M edium sized tumors (up to $2 \mathrm{~cm}$ ) have a spherical cisternal component which is centered at the porus acousticus and a stem that extends into IA C expanding the porus. This gives the appearance of an ice cream cone or mushroom (Fig. 5C). L arger tumors $(2-4 \mathrm{~cm})$ are lobulated or ovoid. Very large tumors usually arise from the cisternal portion of the nerve and may or may not have an intracanalicular component. Such tumors masquerade as meningioma or metastasis. Regardless of size, acoustic schwannomas remain centered on the porus acousticus. M ajority of them show acute angles at the bone-tumor interface in contrast to meningiomas which show obtuse angles. ${ }^{41}$ As the size of the lesion increases, they become more heterogenous with internal necrosis and cystic change. Larger lesions are often associated with extramural (arachnoid) cysts. ${ }^{42}$

On T1-weighted images, they appear isointense or slightly hypointense to pons and hyperintense to CSF. On T2-weighted images, they are hyperintense to pons and isoto hypointense to $\mathrm{CSF}^{43} \mathrm{~A}$ xial $\mathrm{CISS}$ sequence well delineates the tumor, adjacent fine anatomic structures and the fundus of IAC. A coustic schwannomas enhance intensely after injection of gadolinium on T1-weighted images. ${ }^{44}$ Contrast-enhanced M RI has very high sensitivity of nearly $100 \%$ in detecting schwannomas. ${ }^{45}$

\section{Meningioma}

$M$ eningioma is the second most common tumor of CPA. Often there is difficulty in differentiating it from acoustic schwannoma. However, unlike acoustic schwannomas, they are eccentric to porus acousticus, hemispherical lesions with broad-base of the lesion against the dural surface and with obtuse bone tumor angle. ${ }^{41}$ Signal characteristics on M RI also help them to differentiate from other lesions. They are isointense to mildly hypointense to gray matter on T1weighted images and have variable signal intensity on T2weighted imaging which depends on the histopathology of the lesion. If the signal intensity of the lesion is less than or equal to gray matter on T2-weighted images, the most likely diagnosis is meningioma (Figs $6 \mathrm{~A}$ to $\mathrm{C}$ ). ${ }^{46,47}$ Heterogeneity in meningiomas is due to presence of calcifications and cystic change. "Dural tail sign" described for meningioma is not specific and may be found in other tumors, including 
oligodendroglioma, schwannoma, glioblastoma and metastasis. ${ }^{48}$

\section{Epidermoid and Arachnoid Cysts}

It is important to differentiate these two entities as the management differs. B oth are of CSF density, intensity on CT and MRI and do not show postcontrast enhancement. E pidermoid cysts have variable shapes with fine cauliflower like irregularity along their surfaces whereas arachnoid cysts possess smooth surfaces. ${ }^{49}$ Imaging characteristics on certain M R pulse sequences, like diffusion weighted imaging, fluid attenuation inversion recovery (FLAIR) and CISS sequences, help to differentiate arachnoid from epidermoid cyst. E pidermoid cysts appear bright on diffusion-weighted imaging due to restricted diffusion and they are not completely suppressed on FLAIR sequence giving heterogenous appearance in contrast to arachnoid cysts ${ }^{50-52}$ (Figs 7A to D).

\section{Lipoma}

Lipomas are hyperintense on precontrast T1-weighted images. Fat suppression sequences may be used to confirm the presence of fat.

\section{Schwannomas from Other Cranial Nerves}

Nonacoustic schwannomas have similar imaging characteristics as acoustic schwannoma. The precise location of the lesion and evidence of widening of particular neural foramen serves to determine its origin. Trigeminal schwannoma may involve both middle and posterior cranial fossa. It is centered anteromedial to IAC. ${ }^{53}$ Schwannomas from IX, X and XI cranial nerves within the posterior fossa should be considered in the differential diagnosis of acoustic

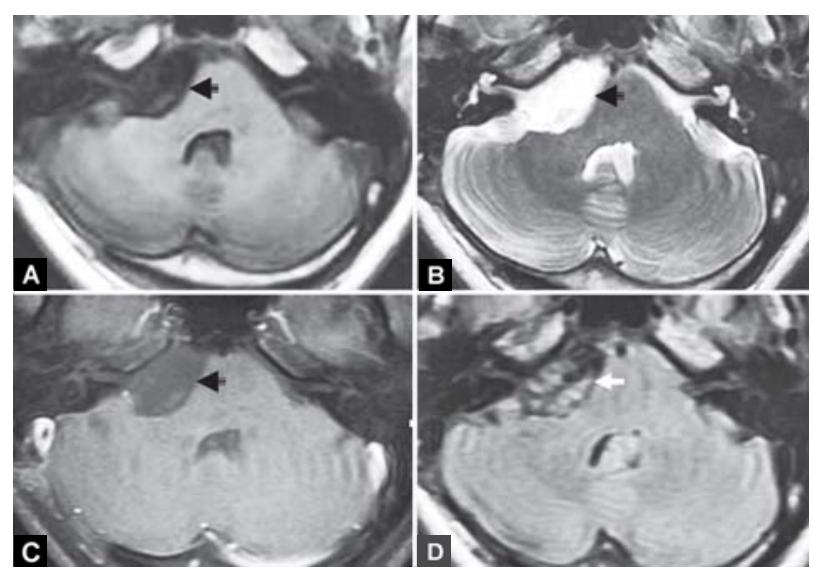

Figs 7A to D: Cerebellopontine angle epidermoid cyst. (A) Axial T1-weighted image, (B) T2-weighted image, (C) contrast-enhanced T1-weighted image. A cystic lesion (open black arrows) is seen with CSF signal intensity on T1 and T2-weighted images with no postcontrast enhancement. The IAC is normal. (D) FLAIR image (white arrow). The lesion is heterogenous with hyperintense foci, enabling differentiation of an epidermoid cyst from an arachnoid cyst schwannomas. Foraminal widening, if evident, aids in making the diagnosis. ${ }^{54}$ This is important as the surgeon avoids the translabyrinthine approach which would otherwise compromise hearing. Facial nerve schwannomas are dealt in the section of facial nerve pathologies.

\section{Vascular Lesions}

Vertebrobasilar dolichoectasia, vascular loops, aneurysms, arteriovenous malformations and hemangiomas are the vascular lesions in CPA.

$\checkmark$ ertebrobasilar dolichoectasia is well demonstrated on $M R I$ as flow void and MR angiography provides more graphic depiction and replaced angiography. ${ }^{55}$ A nterior inferior cerebellar artery, posterior inferior cerebellar atery or the vertebral artery form a loop under, over or between the V II and V III cranial nerves and may produce symptoms, if the compression coincides with the glial Schwann cell junction. New strict criteria have been developed for the diagnosis of neurovascular conflict that in appropriate clinical setting, the vessel should be an artery, site of contact must be the root entry zone with the vessel crossing the nerve perpendicularly, and the nerve must be deviated or indented by the offending vessel or compressed or encased between two or more vessels. ${ }^{56,57}$ Newer M R pulse sequences, like CISS, thin T2-weighted sequences and M R angiography, help to image this condition. ${ }^{58,59}$ As surgical intervention is highly successful, imaging plays a crucial role in the identification of this pathology. A neurysms with patent lumen are seen as flow void whereas thrombosed ones show laminated appearance of varying signal intensities and sometimes a hypointense rim. ${ }^{60}$

Hemangiomas are more hyperintense on T2-weighted images compared to acoustic schwannomas and may invade the adjacent temporal bone. ${ }^{61}$

\section{Jugular Foramen Lesions}

Paragangliomas constitute $90 \%$ of jugular foramen masses. A bout $10 \%$ of lesions include schwannoma of lower cranial nerves, meningioma, primary and metastatic carcinomas, chondrosarcoma and extension from nasopharyngeal carcinoma. These lesions have typical MR imaging characteristics.

\section{Paraganglioma}

Jugulotympanic paragangliomas may arise within middle ear cavity (glomus tympanicum) or from the adventitia of the jugular bulb (glomus jugulare). Due to the proximity of hypotympanum to jugular fossa, large percentage of tumors involve both regions and can present with pul satile tinnitus, hearing loss and retrotympanic masses. ${ }^{62}$ The physician often cannot distinguish both these entities. Thus, detailed imaging examination is crucial. 
Glomus tympanicum tumor is typically seen as intratympanic soft tissue mass al ong the surface of promontory. Confusion arises when the lesion is hol otympanic. However, it typically encases rather than destroying the ossicular chain, thus differentiating from cholesteatoma. Intense gadolinium enhancement on contrast-enhanced MRI helps in further differentiation of these lesions.

Glomus jugulare tumors result in enlargement of jugular foramen. B ony changes due to paraganglioma and the tumor relationships to the bony landmarks are well demonstrated by $\mathrm{CT}$. However, the infral abyrinthine soft tissue extension is better defined by MRI. Thus, both the modalities are complementary. Paragangliomas have unique M R imaging characteristics. Lesions larger than $2 \mathrm{~cm}$ show salt-andpepper pattern of hyperintensity and hypointensity on $\mathrm{T} 1$ and T2-weighted images. ${ }^{63}$ The salt component corresponds to the stromal elements and pepper component to flow voids (Figs $8 \mathrm{~A}$ and $\mathrm{B}$ ). These findings indicate hypervascularity of the lesion and are highly suggestive of paraganglioma. A drop-out effect in the early enhancement pattern demonstrated on dynamic M R imaging helps to differentiate this lesion from other lesions. ${ }^{64}$ Stabilization or reduction in tumor size, reduced enhancement, diminished flow voids and decrease in T2-hyperintnsity indicate local response to radiation therapy. ${ }^{65}$

Numerous pitfalls on M RI have been described. Flowrelated enhancement (bright signal) is possible due to slow flow of the venous blood and enhancement may occur on contrast-enhanced M R images. This is particularly common on the left side owing to the anatomical dominance of right-sided jugular system. This leads to fal se diagnosis of a lesion and unnecessary morbid therapy. If there are absence of clinical findings, further confirmatory data is required to establish the diagnosis. Repeat M R imaging with flow sensitive sequence combined with noncontrast and contrast enhanced CT are recommended. ${ }^{66}$ The signal intensities of

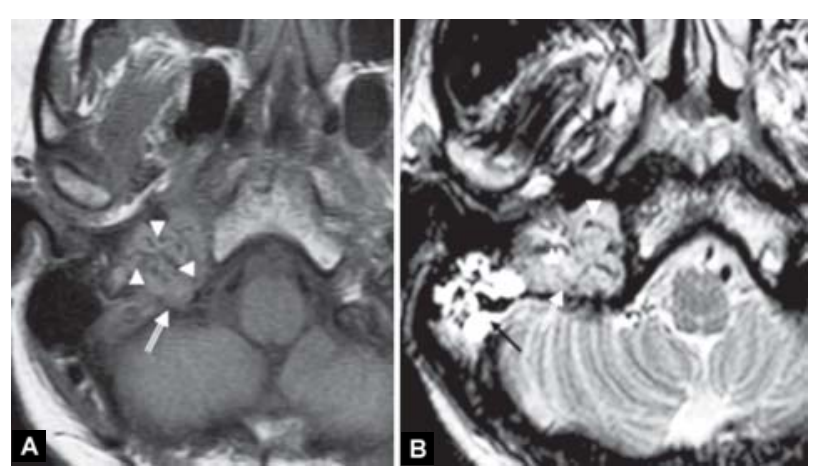

Figs $\mathbf{8 A}$ and $\mathbf{B}$ : Glomus jugulare. (A) axial T1-weighted image and (B) axial T2-weighted image. Soft tissue mass lesion is seen expanding and destroying the jugular fossa. It shows salt- and-pepper pattern of hyperintensity and hypointensity (white open arrows) due to numerous flow voids (white arrow heads) within the lesion. Mastoiditis is seen (black arrow) the tumor must be carefully differentiated from the bone marrow and from mastoid secretions accumulated due to tumor obstruction of tympanic cavity or eustachian tube. It should be noted that the tumor signal intensity is generally more than the cortical bone, aerated air cells, and flowing blood but less than that of bone marrow.

A brief discussion on the role of MRI in patients presenting with pulsatile tinnitus is considered here. A part from paragangliomas, there are numerous arterial, venous and arteriovenous conditions which present as pulsatile tinnitus. ${ }^{67}$

If the tinnitus is suspected to be of venous origin, systemic diseases, intracranial hypertension and lateral sinus stenosis are the major considerations, M R venography and noncontrast M RI of brain are sufficient in most cases. M R venography is used to eval uate the dural sinuses of posterior fossa, to confirm ipsilateral dominant internal jugular vein and to establish contralateral venous drainage in case of intervention. ${ }^{67}$

If it is suggestive of arterial or arteriovenous origin, as indicated by systolic bruit or murmur over head or neck, $M R$ angiography of head and neck and MRI of head will most likely give a diagnosis. M $R$ angiography rules out a stenotic artery or an arteriovenous shunt. ${ }^{68}$ Conventional $M R$ sequences may reveal an abnormal dural sinus and help to evaluate ischemic and hemorrhagic changes of brain. Contrast-enhanced M RA may be considered, if conventional M RA fails to demonstrate a small fistula. ${ }^{69}$ Source images of $M R$ angiography often delineate the transosseous collaterals present within dural arteriovenous fistulae. ${ }^{68}$

M R appearance of jugular schwannoma is similar to that of acoustic schwannoma. M RI best demonstrates the tumor and its extracranial component along the carotid sheath. Jugular meningiomas have similar imaging characteristics as other meningiomas and they typically lack the salt-andpepper pattern of paragangliomas. ${ }^{70}$ Typical M R imaging appearance of chondrosarcoma is the presence of inhomogenous T2-hyperintensities within the lesion. On contrast administration, it shows marked inhomogenous enhancement with components containing small, poorly enhancing or nonenhancing foci. ${ }^{71}$

\section{Petrous Apex Lesions}

Petrous apex mass lesions are principally primary lesions arising within the petrous bone. There can be secondary involvement by lesions of adjacent structures, like CPA, jugular foramen, petroclival junction, geniculate fossa or tympanic cavity. If a lesion is localized within the petrous apex rather than the contiguous structures, one should determine if the lesion is a solid tumor, a cystic expansile lesion or perhaps even a normal variation of the anatomy. 


\section{Normal Variants}

Petrous apex pneumatization can be asymmetric. The marrow fat in the nonpneumatized side appears hyperintense on T1-weighted images which fades in signal intensity on T2-weighted images, paralleling the signal of fat. This is in contrast to pathologic entities, such as cholesteatoma, chol esterol granulomas and most tumors which hyperintense on T2-weighted imaging. However, on fast spin echo, T2-weighted sequences, fat shows significant bright signal. $U$ se of fat suppression sequence resolves the issue (Figs $9 \mathrm{~A}$ and $B$ ). A nother pitfall on $M R I$ is due to unilateral retention of secretions in apical air cells. This appears as hyperintensity on $\mathrm{T} 2$-weighted images and hypointense on T1-weighted images. Both these situations can be clarified by $C T$. If there is no evidence of expansion or erosion of air cells, true tumors or cysts are unlikely. ${ }^{71,72}$

Cystic lesions: Cystic lesions do not show enhancement in the central portion. A thin, smooth peripheral rim enhancement can be seen in some of the lesions.

\section{Cholesterol Granuloma}

Cholesterol granuloma (cyst) is the most common primary petrous apex lesion. ${ }^{73}$ The distinct $M R$ imaging characteristics of this lesion is due to the presence of hemorrhage and cholesterol crystals within it. It appears strongly hyperintense on both $\mathrm{T} 1$ and $\mathrm{T} 2$-w eighted images. A reas of hypointensity representing hemosiderin from previous hemorrhage may be seen. A hypointense rim on both T1 and T2-weighted images may be seen in some of the lesions. Peripheral magnetic susceptibility artifact on gradient echo sequences and central evidence of aliphatic protons on chemical shift imaging is considered almost diagnostic and add specificity to the diagnosis. ${ }^{74}$ I t is crucial to determine the relation of carotid artery, for which CT is the preferred imaging modality.

\section{Petrous Carotid Artery Aneurysms}

It is extremely important to consider petrous carotid artery aneurysm in the differential diagnosis of expansile petrous

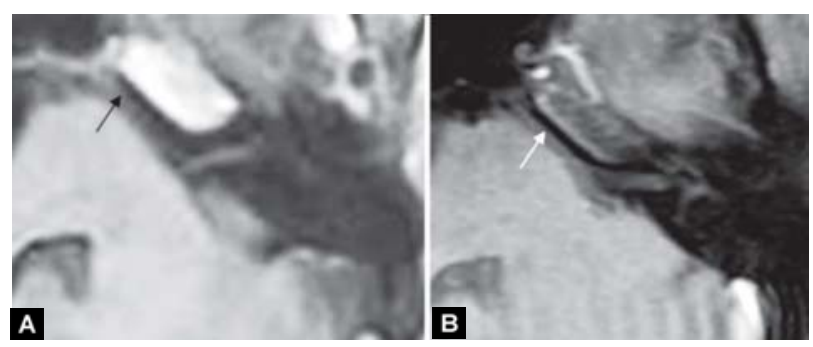

Figs 9A and B: Pneumatization of petrous apex. (A) axial T1-weighted image and (B) fat-suppressed T1-weighted image. Hyperintensity in the petrous apex due to marrow fat (black arrow) can mimic a lesion. Fat suppression sequence clarifies the issue as the fat gets suppressed (white arrow) lesions. ${ }^{75}$ The M R appearance is variable depending on the extent of thrombus within the aneurysm. The patent arterial lumen is identified as a flow void and laminated mural thrombus shows varying signal intensities. ${ }^{76}$ Demonstration of intact cortical wall of the carotid canal is the key finding which helps to exclude this important diagnosis. There is definitely an overlap between cholesterol cyst and carotid aneurysm. ${ }^{72}$

Epidermoid cysts (congenital cholesteatomas) and mucoceles have very similar appearance on M R imaging. They appear hypointense on T1-weighted images and hyperintense on T2-weighted images. A rachnoid cysts have been identified in the petrous apex and these follow CSF signal intensity on all M R sequences.

$M$ eningoceles from M eckel's cave can mimic expansile cystic lesions of petrous apex and they follow CSF signal intensity on various $M R$ pulse sequences.

Solid or noncystic lesions: If there is enhancement of central portion of the lesion, it is considered as a solid lesion. $M$ eningiomas and chondrosarcomas are the common solid tumors involving the region of petrous apex. Imaging findings of these lesions have al ready been described.

\section{Facial Nerve Lesions}

Knowledge and good understanding of the function and anatomical course of facial nerve and its branches helps to localize the exact site of injury and thus helps to narrow the search for pathology and tailor the imaging protocol. This enables the surgeon to come up with a good treatment plan.

Facial nerve is divided into cisternal segment, intratemporal segment and peripheral segment. For the purpose of this article, cisternal and intratemporal segments are considered and the relevant anatomy pertaining to $M R$ imaging is discussed. The reader can refer to an anatomy book for detailed anatomy. Facial nerve exits at the lower border of the pons at the recess between the olive and superior cerebellar peduncle. It courses through cerebellopontine angle cistern (cisternal segment) to enter the porus acousticus of the IAC along with vestibulocochlear nerve. IAC is divided into four quadrants by crista falciformis, a horizontal bony crest and by a vertically oriented structure referred to as Bill's bar. ${ }^{77}$ W ithin the IAC, facial nerve is superior to cochlear nerve and anterior to the superior vestibular nerve. Thus, facial nervelies in the anterosuperior quadrant, cochlear nerve in the anteroinferior quadrant and vestibular nerves in the posterior quadrants. ${ }^{78}$ The mediolateral orientation of IACs is such that the porus is more superior on coronal images. IA C s are oriented nearly perpendicular to the sagittal plane of the skull. High resolution M R images in oblique sagittal plane perpendicular to the plane of IA Cs can demonstrate all the four nerves in cross section and accurate assessment of their diameters can be made. $^{7}$ 
The intratemporal segment of facial nerve is further divided into labyrinthine, tympanic and mastoid segments. The labyrinthine segment courses anterolaterally from the fundus of the IAC to terminate at the geniculate ganglion. This is the location where there is a crossroad of four nerve canals. They include labyrinthine segment, tympanic segment of facial nerve, lesser petrosal and greater petrosal nerves. Nerve canals containing both the petrosal nerves may be seen on routine MRI but are better appreciated if enlarged in pathologic states, such as antegrade or retrograde spread of a tumor. Due to the presence of a circumneural venous plexus surrounding intratemporal facial nerve, normal enhancement can be seen particularly in the region of the geniculate ganglion. ${ }^{79}$ Due to great degree of variation in the enhancement, pathological enhancement of the nerve should be considered only when there is concurrent nodularity or obvious expansion. Distal intracanalicular and labyrinthine portions do not show physiological enhancement due to absence of perineural plexus. Thus, enhancement in these portions may indicate pathology. Criteria for defining pathological enhancement have not been fully determined. ${ }^{80}$

The choice of imaging modality depends on the portion of the facial nerve being studied and the clinical indication. The brainstem nuclei, the cisternal segment and the intracanalicular segments are better evaluated with MRI. HRCT is superior to image the tympanic segment. The mastoid segment may be evaluated with either modality, $M R I$ is favored to image the mastoid portion as lack of mastoid pneumatization limits the use of $\mathrm{HRCT},{ }^{57}$ If the origin of a peripheral facial palsy cannot be localized clinically, MRI is the preferred imaging modality. The imaging evaluation should include not only the temporal bone but also the brainstem, CPA and the parotid gland in such patients. . $^{5,71}$

Facial nerve injury can occur due to inflammatory, neoplastic, traumatic etiology or infiltration by lesions of the temporal bone.

\section{Inflammatory Conditions}

B ell's palsy is the classic inflammatory neuropathy of the facial nerve. The diagnosis of this condition is based on clinical findings. M ost patients do not require any imaging evaluation. M RI is indicated in atypical B ell's palsy: W hen it persists for more than 4 months, with multiple cranial involvement, with recurrent symptoms or when the onset is subacute. ${ }^{81}$ Contrast-enhanced M RI shows enhancement of the nerve with or without discernible enlargement (Figs 10A and $B$ ). A s previously described, mere enhancement of the nerve does not indicate the pathology, unless intracanal icular and cisternal segements show enhancement. ${ }^{79} \mathrm{H}$ owever, the classical description of B ell's palsy is uniform enhancement

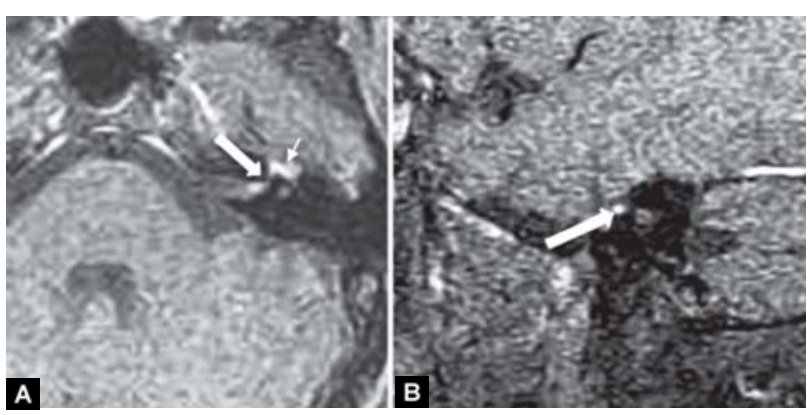

Figs 10A and B: Bell's palsy. (A) Axial post contrast T1-weighted image, (B) Oblique sagittal post contrast T1-weighted image. There is enhancement of intracanalicular portion (solid white arrows) and proximal intratemporal portion (small white arrow) of facial nerve. The location of facial nerve in the anterosuperior quadrant of IAC is well demonstrated on oblique sagittal image

of the intratemporal segment with little or no enlargement and no nodularity. ${ }^{82} \mathrm{M} \mathrm{RI}$ in Ramsay H unt syndrome shows enhancement of acoustic nerve and membranous labyrinth in addition to facial nerve thus allowing precise diagnosis. ${ }^{83}$

A s leptomeninges follow the seventh and eighth cranial nerves into IAC, basal meningitis due to tuberculosis or sarcoidosis can involve the facial nerve, especially the cisternal and intracanalicular portions. ${ }^{83,84}$ O ther inflammatory processes of adjacent temporal bone can also involve the facial nerve.

\section{Neoplastic Conditions}

Primary neoplasms of facial nerve include schwannoma, hemangioma, epidermoid cyst. Other rare tumors are choristoma and primary paraganglioma.

Schwannomas can arise from any portion of the facial nerve with geniculate ganglion being the most common site of involvement. Intracanalicular and cisternal facial schwannomas have similar appearance to acoustic schwannomas as previously described. Imaging helps to distinguish facial and vestibular schwannomas, based on the anterior or posterior location within the cistern and IA C. However, this can only be done with certainity, if there is extension of the lesion along the intratemporal portion of the nerve. ${ }^{4}$ Schwannomas cause smooth expansion of facial canal causing sharply defined bony margins.

Hemangiomas predominantly occur at the geniculate ganglion, next most often in the IAC, and least common at the posterior genu. Tumors in the IAC are well demonstrated by M RI. ${ }^{85}$ Geniculate ganglion lesions are better seen on CT than noncontrast MRI. These may show intratumoral bony spicules and irregular margins, best seen on CT. They are iso to mildly hyperintense on T1-weighted images and markedly hyperintense on T2-weighted images, more than the typical schwannoma. Inhomogenous signal intensities corresponding to bony spicules may be seen on M RI. Thin 
sections and use of contrast-enhanced M RI may improve the MR imaging results. ${ }^{71}$

Tumorsfrom adjacentstructures may secondarily involve the facial nerve, most common being jugular foramen paraganglioma. Other tumors include endolymphatic sac tumor, tumors of parotid, external auditory canal and on rare ocacasions systemic malignancies.

\section{Traumatic Lesions}

Facial nerve paralysis is associated with 25 to $70 \%$ of temporal bone fractures. ${ }^{86}$ It is more commonly with transverse than with longitudinal fractures.$^{87}$ Though HRCT demonstrates the exact course of the fracture and its relation to bony facial canal, the nerve itself cannot be seen. M RI scores over CT, as it hel ps in visual ization of the nerve itself, allowing assessment of its size and the enhancement pattern. A bnormal enhancement of the nerve is al most al ways seen in the distal intracanalicular segment and commonly seen in the labyrinthine segment, proximal tympanic segment and geniculate ganglion. ${ }^{83}$ This enhancement is secondary to disruption of blood nerve barrier. There is no correlation between enhancement and the degree of nerve injury. Prolonged abnormal enhancement of the nerve can be seen, especially the distal intracanalicular segment which may persist for up to 2 years. ${ }^{87}$ Thin noncontrast T1-weighted images demonstrate hematoma formation.

\section{Postoperative Temporal Bone Imaging}

High-resolution CT is an excellent tool for evaluating postoperative middle ear and mastoid as it provides striking contrast between residual or recurrent debris and the aircontaining cavity. However, MRI is indicated in certain situations.

$M R I$ is strongly recommended when a postoperative defect is detected in the region of the tegmen or sinus plate. A soft tissue mass protruding through a defect in tegmen tympani may represent a meningocele or meningoencephalocele. ${ }^{54} \mathrm{MRI}$ easily identifies the presence or absence of brain tissue within the mass and confirms contiguity of the mass with adjacent brain. MRI provides crucial information regarding cerebellum and sigmoid sinus in patients with bony defects. ${ }^{88}$

Numerous varieties of prostheses have been developed for ossicul oplasty and ossicular reconstruction over the past few decades. A number of materials are used for manufacturing these prostheses. The reader should be aware that a recent study concluded that most of these devices are nonferromagnetic, and M R imaging is generally considered safe for these patients. Only those patients who underwent surgery many years ago with a device not included in this study are exceptional. ${ }^{89}$
$M R I$ is generally prevented in individuals with cochlear implants as it may cause demagnetization of the internal magnet of the implant leading to its dysfunction or there can be possibility of injuring the patient. Significant artifacts occur due to this internal magnet. Studies have been conducted to determine whether patients with a cochlear implant can safely undergo an M R examination. Highly specific guidelines have been generated in order to peform a safe M R examination in these patients. Some of the devices require the use of low magnetic field strength $M R$ systems. Some may require removal of the internal magnet and external hardware before performing M R examination while others have been proven safe even without removal of the internal magnet. ${ }^{90}$

Postoperative evaluation of CPA and IAC is often challenging. Thin, linear enhancement within and adjacent to the IA C is considered anormal finding in the postoperative setting and itis attributed to dural (pachymeningeal) irritation. This may persist over many months. Follow-up imaging at specified interval is recommended under these circumstances. ${ }^{91,92}$ The translabyrinthine approach used in the removal of acoustic tumors may pose a special problem in the postoperativeeval uation as these defects are commonly packed with fat. U se of fat suppression sequence or a precontrast T 1-weighted sequence helps to distinguish the fat from enhancing debris and tumor..$^{57}$

\section{Intraoperative MRI and Neuronavigational Techniques}

A number of systems based on preoperatively acquired data have been developed over the past few years to enhance surgical navigation. It provides intraoperative orientation of the lesion, its relation to adjacent structures and helps to plan the precise surgical approach. Combining the functional data provided by functional MRI and magnetoencephalography (MEG) with neuronavigation prevents injury to eloquent areas of brain during surgery. Advancements in CT and MRI fusion imaging enable the surgeon to take advantage of the bony detail provided by $\mathrm{CT}$ and the soft tissue detail provided by the MRI.

However, during surgical exposure and resection of the lesion, the three-dimensional relationship of the lesion to adjacent structures is lost due to brain shift. This leads to the development of intraoperative imaging. A $n$ intraoperative MRI enables management of patients with complex skull base lesions by performing radical resection of the lesions. The updates of neuronavigation with the intraoperative images hel $p$ to compensate for brain shift. ${ }^{93-95}$

\section{CONCLUSION}

To conclude, M RI plays a crucial role in imaging of lateral skull base lesions. MRI improves detection and understanding of pathologies which may alter the treatment plan. The structural and functional information provided 
by $\mathrm{M}$ RI and intraoperative technique enable the surgeon to perform safe surgery with minimal morbidity. M RI is also useful in post-treatment evaluation of patients. Though it is superior to CT in demonstrating soft tissue pathologies, CT remains the modality of choice to evaluate the bony abnormalities. Thus, both these techniques can be complementary and not competitive in evaluating skull base lesions as one method fills in details which are absent in the other.

\section{REFERENCES}

1. Sanna M, Saleh EA, A lexandria, et al. A tlas of microsurgery of the lateral skull base (2nd ed). Thieme Medical Publishers 2008; 13-24.

2. Som PM , Curtin HD. Head and neck imaging (4th ed). St L ouis: M osby 2003;789-864.

3. Som PM , Curtin HD. Head and neck imaging (4th ed). St L ouis: M osby 2003:1093-108.

4. Swartz JD, Harnsberger HR (Eds). I maging of the temporal bone (3rd ed). Thieme M edical Publishers 1998.

5. Santosh SG, Shailendra RM, M ilind VK, Nitin S. Pictorial review of $\mathrm{M} R \mathrm{RI} / \mathrm{CT}$ scan in congenital temporal bone anomalies in patients for cochlear implant. Indian J Radiol Imaging 2009;19:99-106.

6. Som PM , Curtin HD. Head and neck imaging (4th ed). St L ouis: M osby 2003;1109-71.

7. Kim HS, Kim DI, Chung IH. Topographical relationship of the facial and vestibulocochlear nerves in the subarachnoid space and internal auditory canal. AJNR A m J Neuroradiol 1998;19:1155-61.

8. Glastonbury CM, Davidson HC, Harnsberger HR. Head and neck imaging findings of cochlear nerve deficiency. A J N R A m J Neuroradiol 2002;23:635-43.

9. Nadol JB J r, Xu W Z. Diameter of the cochlear nerve in deaf humans: Implications for cochlear implantation. Ann Otol Rhinol L aryngol 1992;101:988-93.

10. Y likoski J, Savolainen S. The cochlear nerve in various forms of deafness. A cta Otolaryngol 1984;98:773-81.

11. M afee M F, C harletta $D, K$ umar A, B elmont $H$. L arge vestibular aqueduct and congenital sensorineural hearing loss. AJNR A m J Neuroradiol 1992;13:805-19.

12. Phelps $P$, et al. Radiologic manifestations of the ear in Pendred syndrome. Clin Radiol 1998;53:268-73.

13. Casselman JW, Kuhweide R, D eimling $M$, et al. Constructive interference in steady state-3DFT M R imaging of the inner ear and cerebellopontine angle. AJNR Am J Neuroradiol 1993;14:47-57.

14. V ogl TJ, Bergman C, V illringer A, et al. Dural sinus thrombosis: $V$ alue of venous $M R$ angiography for diagnosis and follow-up. AJR 1994;162(5):1191-98.

15. Kelley KE, Jackler RIC, Dillon W P. Diagnosis of septic sinus thrombosis resonance imaging. Otolaryngol Head Neck Surg $1991 ; 105(4): 617-24$.

16. M anzione J, Newman GC, Shapiro A, et al. Diffusion- and perfusion-weighted $M R$ imaging of dural sinus thrombosis. AJ NR A m J Neuroradiol 2000;21(1):68-73.

17. Som PM , Curtin HD. Head and neck imaging (4th ed). St L ouis: M osby 2003;1173-229.
18. M artin N, Sterkers $\mathrm{O}, \mathrm{N}$ ahum $\mathrm{H}$. Chronic inflammatory disease of the middle ear cavities: Gadolinium-DTPA enhanced MR imaging. Radiology 1990;176:399-405.

19. M artin N, Sterkers $0, M$ ompoint $D$, et al. Cholesterol granulomas of the middle ear cavities: M R imaging. Radiology 1989;172:521-25.

20. Latack JT, Graham M D, I semink JC, et al. Giant cholesterol cysts of the petrous apex. AJNR Am J Neuroradiol 1985;6: 409-17.

21. Mafee MF. MR and CT in the evaluation of acquired and congenital cholesteatomas of the temporal bone. J Otolaryngol 1993;22:239-48.

22. M ark AS, Fitzgerald D. Segmental enhancement of the cochlear on contrast-enhanced M RI: Correlation with the frequency of hearing loss and possible sign of perilymphatic fistula and auto-immune labyrinthitis. A J R A m J Neuroradiol 1993;14: 991-96.

23. Weissman JR, Cumin HD, Hirsch B, et al. High signal from the otic labyrinth on unenhanced magnetic resonance imaging. AJNR A m J Neuroradiol 1992;13:1183-87.

24. Casselman JW, K uhw eide R, A mpe W, et al. Pathology of the membranous labyrinth: Comparison of T1- and T2-weighted and gadolinium-enhanced spin-echo and 3DFT-CISS imaging. AJNR A m J Neuroradiol 1993;14(1):59-69.

25. Casselman JW, M ajoor M H, A lbers FW. M R of the inner ear in patients with Cogan syndrome. AJNR Am J Neuroradiol 1994;15(1):131-38.

26. Grandis J R, Curtin HD, Y u V L. Necrotizing (malignant) external otitis: Prospective comparison of $C T$ and $M R$ imaging in diagnosis and follow-up. Radiology 1995;196(2):499-504.

27. Seabold JE, Simonson TM, W eber PC, et al. Cranial osteomyelitis: Diagnosis and follow-up with In-111 white blood cell and Tc-99m methylene diphosphonate bone SPECT, CT and M R imaging. Radiology 1995;196(3):779-88.

28. Som PM , Curtin HD. Head and neck imaging (4th ed). St L ouis: M osby 2003;1230-44.

29. Sartoretti-Schefer S, Scherler M, W ichmann W, et al. Contrastenhanced M R I of the facial nerve in patients with post-traumatic peripheral facial nerve palsy. AJNR Am J Neuroradiol 1997;18:1115-22.

30. Zimmerman RA, Bilaniuk LT, Hackney DB, et al. Magnetic resonance imaging in temporal bone fractures. N euroradiology 1987;29:246-51.

31. Whitehead RE, MacD onald CB, M elhem ER, M CM ahon $L$. Spontaneous labyrinthine hemorrhage in sickle cell disease. AJNR A m J Neuroradiol 1998;19:1437-40.

32. Jane NN, Laureno R, M ark AS, et al. Deafness after bilateral midbrain contusion: A correlation of magnetic resonance imaging with auditory brainstem evoked responses. Neurosurgery 1991;29:1101.

33. Valavanis $A$, Schubiger $O, N$ aidich $T$. Clinical imaging of the cerebellopontine angle. Berlin: Springer-V erlag 1986.

34. Bebin J. Pathophysiology of acoustic tumors. Diagnosis. Baltimore: University Park Press 1979:1.

35. Brow R. Pre- and postoperative management of the acoustic tumor patient. M anagement. Baltimore: University Park Press 1979:2.

36. Selesnick SH, Jackler RK. C linical manifestations and audiologic diagnosis of acoustic neuromas. Otolaryngol Clin North Am 1992;25(3):521-51.

37. Zealley IA, Cooper RC, Clifford KM, et al. M RI screening for acoustic neuroma: A comparison of fast spin echo and contrast enhanced imaging in 1233 patients. Br J Radiol 2000;73: 242-47. 
38. Somers T, Casselman J, de Ceulaer G, G ovaerts P, Offeciers E. Prognostic value of magnetic resonance imaging findings in hearing preservation surgery for vestibular schwannoma. 0 tol Neurotol 2001;22(1):87-94.

39. Dubrulle $F$, Ernst $O$, V incent $C$, et al. Cochlear fossa enhancement at MR evaluation of vestibular schwannoma: Correlation with success at hearing-preservation surgery. Radiology 2000;215(2):458-62.

40. Solti-Bohman LG, M agaram DL, Lo WW, et al. Gas-CT cisternography for detection of small acoustic nerve tumors. Radiology 1984;150(2):403-07.

41. Valavanis A, Schubiger 0 , Hayek J, Pouliadis G. CT of meningiomas on the posterior surface of the petrous bone. Neuroradiology 1981;22(3):111-21.

42. Tali ET, Y uh WT, N guyen HD, et al. Cystic acoustic schwannomas: M R characteristics. A J R 1993;14(5):1241-47.

43. Press $G A$, Hesselink JR. M R imaging of cerebell opontine angle and internal auditory canal lesions at $1.5 \mathrm{~T}$. AJR 1988;150(6):1371-81.

44. Curati $W L$, Graif $M$, Kingsley DP, Niendorf HP, Y oung IR. A coustic neuromas: Gd-DTPA enhancement in MR imaging. Radiology 1986;158(2):447-51.

45. Curtin HD. Rule out eighth nerve tumor: Contrast-enhanced T1-weighted or high-resolution T2-weighted M R. A JNR 1997;18(10):1834-38.

46. Elster AD, Challa VR, Gilbert TH, Richardson DN, Contento JC. M eningiomas: M R and histopathologic features. Radiology 1989;170(3 Pt 1):857-62.

47. Gentry LR, J acoby CG, Turski PA, et al. Cerebellopontine anglepetromastoid mass lesions: Comparative study of diagnosis with M R imaging and CT. Radiology 1987;162(2):513-20.

48. Wilms G, Lammens $M, M$ archal $G$, et al. Prominent dural enhancement adjacent to nonmeningiomatous mal ignant lesions on contrast-enhanced MR images. AJNR A m J Neuroradiol 1991;12(4):761-64.

49. B erger M S, W ilson CB. E pidermoid cysts of the posterior fossa. J Neurosurg 1985;62(2):214-19.

50. Tsuruda J S, Chew WM, M oseley ME, Norman D. Diffusionweighted $M R$ imaging of the brain: Value of differentiating between extra-axial cysts and epidermoid tumors. AJR 1990;155(5):1059-65; discussion 1066-68.

51. M urakami $\mathrm{N}, \mathrm{M}$ atsushima $\mathrm{T}, \mathrm{K}$ uba $\mathrm{H}$, et al. Combining steadystate constructive interference and diffusion-weighted magnetic resonance imaging in the surgical treatment of epidermoid tumors. Neurosurg Rev 1999;22(2-3):159-62.

52. Gizewski E. Epidermoid or arachnoid cyst: CISS, FLAIR and diffusion images as solution of the diagnostic dilemma. R ofo Fortschr Geb Rontgenstr Neuen Bildgeb Verfahr 2001;173(1):77-78.

53. K apila A, Chakeres DW, B lanco E. The M eckel cave: Computed tomographic study. Part I: Normal anatomy; Part II: Pathology. Radiology 1984;152(2):425-33.

54. Neely JG. Reversible compression neuropathy of the eighth cranial nerve from a large jugular foramen schwannoma. A rch Otolaryngol 1979;105(9):555-60.

55. M eaney JF, Miles JB, Nixon TE, Whitehouse GH, Ballantyne ES, Eldridge PR. V ascular contact with the fifth cranial nerve at the pons in patients with trigeminal neural gia: Detection with 3D FISP imaging. AJR 1994;163(6):1447-52.

56. Borges A, Casselman J. Imaging the cranial nerves: Part II: Primary and secondary neoplastic conditions and neurovascular conflicts. Eur Radiol 2007;17:2332-44.
57. Swartz JD, Harnsberger HR, M ukherji SK. The temporal bone contemporary diagnostic dilemmas. Radiol Clin North A m 1998;36:819-53.

58. Tan EK, Chan LL, Lim SH, Lim WE, K hoo JB, Tan K P. Role of magnetic resonance imaging and magnetic resonance angiography in patients with hemifacial spasm. A nn A cad M ed Singapore 1999;28(2):169-73.

59. Y amakami I, K obayashi E, Hirai S, Y amaura A. Preoperative assessment of trigeminal neural gia and hemifacial spasm using constructive interference in steady state three-dimensional fourier transformation magnetic resonance imaging. Neurol M ed Chir (Tokyo) 2000;40(11):545-55; discussion 555-56.

60. OIsen WL, Brant-Zawadzki M, Hodes J, Norman D, Newton TH. Giant intracranial aneurysms: MR imaging. Radiology 1987;163(2):431-35.

61. Lo WW, Shelton C, Waluch V, et al. Intratemporal vascular tumors: Detection with $\mathrm{CT}$ and MR imaging. Radiology 1989;171(2):445-48

62. Lo WW, Solti-B ohman LG. High-resolution CT of the jugular foramen: A natomy and vascular variants and anomalies. Radiology 1984;150(3):743-47.

63. Olsen $W L$, Dillon WP, Kelly $W M$, et al. MR imaging of paragangliomas. AJR 1987;148(1):201-04.

64. Vogl TJ, Mack MG, Juergens $M$, et al. Skull base tumors: Gadodiamide injection-enhanced M R imaging-drop-out effect in the early enhancement pattern of paragangliomas versus different tumors. Radiology 1993;188(2):339-46.

65. M ukherji SK, Kasper ME, Tart RP, M ancuso AA. Irradiated paragangliomas of the head and neck: $C T$ and $M R$ appearance. A JNR A m J Neuroradiol 1994;15(2):357-63.

66. Widick M H, Haynes DS, Jackson G, et al. Slow flow phenomena in magnetic resonance imaging of the jugular bulb masquerading as skull base neoplasms. A m J Otology 1996;17:648-52.

67. Som PM , C urtin HD. Head and neck I maging (4th ed). St L ouis: M osby 2003;1361-74.

68. Dietz RR, Davis WL, H arnsberger $H R$, et al. MR imaging and $M R$ angiography in the evaluation of pulsatile tinnitus. AJNR A m J Neuroradiol 1994;15:879-89.

69. Chen JC, TsurudaJ S, Hal bach W. Suspected dural arteriovenous fistula: Results with screening M R angiography in seven patients. Radiology 1992;183:265-71.

70. Spagnoli MV, Goldberg HI, Grossman RI, et al. Intracranial meningiomas: High-field MR imaging. Radiology 1986; 161(2):369-75.

71. Som PM , C urtin HD. Head and neck I maging (4th ed). St L ouis: M osby 2003;1275-360.

72. Curtin HD, Chavali R. Imaging of the skull base. Radiol Clin North A m 1998;36(5):801-17.

73. L atack JT, Graham M D, K emink JL, K nakeJE. Giant cholesterol cysts of the petrous apex: Radiologic features. AJNR Am J Neuroradiol 1985;6(3):409-13.

74. Greenberg J J, O ot RF, Wismer GL, et al. Cholesterol granuloma of the petrous apex: MR and CT evaluation. AJNR A m J Neuroradiol 1988;9(6):1205-14.

75. Kudo S, Colley DP. Multiple intrapetrous aneurysms of the internal carotid artery. AJNR Am J Neuroradiol 1983;4(5): 1119-21.

76. Atlas SW, G rossman RI, Goldberg HI, et al. Partially thrombosed giant intracranial aneurysms: Correlation of M R and pathologic findings. Radiology 1987;162:111-14.

77. Jackler RK, B rackmann DE: N euro-otology. St L ouis: M osbyY earbook 1994. 
78. Swartz JD, Daniels DL, Harnsberger, et al. Hearing II: The retrocochlear auditory pathway. AJNR Am J Neuroradiol 1996;17:1479-81.

79. Gebarski SS, Telian SA, Niparko JK. Enhancement along the normal facial nerve and facial canal: M R imaging and anatomic correlation. Radiology 1992;183:384-91.

80. K inoshita T, Ishii K, O kitsu T, O udera T, Ogawa T. Facial nerve palsy: Evaluation by contrast-enhanced M R imaging. Clin Radiol 2001;56:926-32.

81. Smouha EE, Coyle K, Shkri S. Facial nerve palsy in Lyme disease evaluation of clinical diagnostic criteria. Am J Otol 1997;18:257-61.

82. Davidson HC. Imaging of the temporal bone. Magn Reson Imaging Clin N A m 2002;10:573-613.

83. Chan EH, Tan HM, Tan TY. Facial palsy from temporal bone lesions. A nn A cad M ed Singapore 2005;34:322-29.

84. Cinnamon J, Sharma M, Gray $D$, et al. Neuroimaging of meningeal disease. Semin UItrasound CT M R 1994;15:466-98.

85. WW Lo, Shelton C, Waluch V, et al. Intratemporal vascular tumors: Detection with $\mathrm{CT}$ and $\mathrm{MR}$ imaging. Radiology 1989;171(2):445-48.

86. B rodie HA, Thompson TC. M anagement of complications from 820 temporal bone fractures. A m J Otol 1997;18:188-97.

87. Sartoretti-Schefer $S$, Scherler $M, W$ ichmann $W, V$ alavanis $A$. Contrast-enhancement M R of the facial nerve in patients with post-traumatic peripheral facial nerve palsy. AJNR Am J Neuroradiol 1997;18:1115-25.

88. $M$ artin $N$, Sterkers $O, M$ urat $M$, et al. B rain herniation into the middle ear cavity: M R imaging. Neuroradiology 1989;31: 184-86.

89. Shellock FG, Schatz CJ. M etallic otologic implants: In vitro assessment of ferromagnetism at 1.5 T. A J NR A m J Neuroradiol 1991;12(2):279-81.

90. Shellock FG, Shellock R and D Services, Inc. ; 2011. A vailable at: http://www.mrisafety.com.

91. M ueller DP, Gantz BJ, Dolan KD. Gadolinium-enhanced M R of the postoperative internal auditory canal following acoustic neuroma resection via a middle cranial fossa approach. A JNR A m J Neuroradiol 1992;13:197-200.

92. Weissman JL, Hirsch BE, Fukui M B, Rudy TE. The evolving appearance of structures in the internal auditory canal after removal of an acoustic neuroma. AJNR Am J Neuroradiol 1997;18:313-23.

93. A bdullah Al-akayleh. A pplication of neuronavigation in neurosurgery at King Hussein M edical Center, J ordan. Rawal M edical Journal J an-Jul 2010;35(1):91-94.

94. Dort JC, Sutherland GR. Intraoperative magnetic resonance imaging for skull base surgery. Laryngoscope 2001;111: 1570-75.

95. Schlosser RJ, B olger WE. I mage-guided procedures of the skull base. Otolaryngol Clin N A m 2005;38:483-90. 